\title{
Implantable cardioverter defibrillator endocarditis caused by Klebsiella pneumoniae complicated by liver abscess and septic pulmonary embolism
}

\author{
Ilaria Izzo, ${ }^{1}$ Stefano Ettori, ${ }^{1}$ Paolo Colombini, ${ }^{1}$ Marco Cannata, ${ }^{2}$ Adriano Pagani, ${ }^{1}$ Daniele Bella ${ }^{1}$ \\ ${ }^{1}$ UO Medicina Interna, AO Mellino Mellini, Chiari (BS); ${ }^{2}$ Università degli Studi di Brescia, Brescia, Italy
}

\begin{abstract}
A 63-year old diabetic male patient carrying an implantable cardioverter defibrillator (ICD) was hospitalized with a 7day history of fever, notwithstanding an antibiotic therapy. The white-blood cell count was $11,000 / \mathrm{mm}^{3}$, the platelet count was $135,000 / \mathrm{mm}^{3}$ and C-reactive protein (CRP) $13 \mathrm{mg} / \mathrm{dL}$. Chest X-rays showed right infiltrates. Ceftriaxone was started. Defervescence was rapid, but CRP was still $12 \mathrm{mg} / \mathrm{dL}$ after 6 days. A trans-thoracic ecochacardiogram (TTE) incidentally showed a liver hypoechoic lesion. A computed tomography scan revealed bilateral cavitated lung nodules and a large liver abscess. Klebsiella pneumoniae was isolated in blood cultures and TTE showed ICD endocarditis and a patent foramen ovalis. Levofloxacin and imipenem/cilastatin were started. The liver abscess was drained. After 30 days, the ICD was removed and re-implanted. At discharge, blood tests were within the normal range and the patient was asymptomatic. Follow up showed improvement of lung and hepatic lesions. To our knowledge, this is the second reported case of K. pneumoniae infective endocarditis with multiple septic emboli. Endocarditis should be suspected in presence of fever after the device implantation, in particular if risk factors are present.
\end{abstract}

Correspondence: Ilaria Izzo, UO di Medicina Interna, AO Mellino Mellini, via Mazzini 3, 25032, Chiari (BS), Italy. Tel.: +39.030.710210.

E-mail: izzo.ilaria@hotmail.it

Key words: implatable cardioverter defibrillator, endocarditis, Klebsiella pneumoniae, liver abscess.

Contributions: the authors contributed equally.

Acknowledgments: we are grateful to the patient who gave his consent to the publication of this study.

Conflict of interests: the authors declare no potential conflict of interests.

Conference presentation: this paper was presented as poster communication at the $17^{\text {th }}$ Congresso Nazionale FADOI, Giardini Naxos, May 11-14, 2013.

Received for publication: 10 July 2013.

Revision received: 4 September 2013.

Accepted for publication: 16 September 2013.

This work is licensed under a Creative Commons Attribution NonCommercial 3.0 License (CC BY-NC 3.0).

CCopyright I. Izzo et al., 2014

Licensee PAGEPress, Italy

Italian Journal of Medicine 2014; 8:124-126

doi:10.4081/itjm.2013.407

\section{Introduction}

Implantation of cardiac devices is nowadays a routine procedure in cardiovascular medicine. ${ }^{1}$ Cardiac device endocarditis (CDE) has emerged as a serious complication of electrotherapy with a reported incidence between $0.06 \%$ and $7 \% .{ }^{2,3}$ Staphylococcus epidermidis and Staphylococcus aureus are the etiological agents in $70 \%$ to $90 \%$ cases of $\mathrm{CDE}$ and have been extensively studied in the past, while non-staphylococcal organisms have been reported only in case reports or small series. ${ }^{4}$ In particular, Gram negative bacteria are infrequent etiological agents of cardiovascular implantable electronic device infections. ${ }^{1}$ Klebsiella pneumoniae endocarditis is a rare entity with a reported poor prognosis and high mortality. ${ }^{5}$ Rivero et al. reported the first case of liver abscess caused by K. pneumoniae associated with infective endocarditis. ${ }^{6}$

\section{Case Report}

We report the case of a 63-years old man, who was admitted to our hospital with a 7-day history of fever, notwithstanding an empiric antibiotic therapy (amoxicillin/clavulanate $1 \mathrm{~g}$ bis in die per os). The patient had a history of diabetes mellitus, hypertension and was a carrier of an implantable cardioverter dual-chamber defibrillator (ICD) for a ventricular fibrillation episode. At admission, his vital signs were as follows: temperature $38.4^{\circ} \mathrm{C}$, heart rate 80 beats $/ \mathrm{min}$, blood pressure 
$145 / 80 \mathrm{mmHg}$ and oxygen saturation $97 \%$ in room air. Wheezes were present at chest auscultation; the abdomen was soft, with no significant tenderness, hepatomegaly was reported. The rest of the exam was unremarkable. White-blood cell count was $11,000 / \mathrm{mm}^{3}$ and platelet count was $135,000 / \mathrm{mm}^{3}$ with C-reactive protein $(\mathrm{CRP})$ at $13 \mathrm{mg} / \mathrm{dL}$ (normal range $<0.50$ $\mathrm{mg} / \mathrm{dL}$ ). Liver function tests were normal, the urine analysis was unremarkable. His chest X-rays revealed right lobar infiltrates. Intravenous ceftriaxone $2 \mathrm{~g}$ was started as empiric antibiotic therapy for community-acquired pneumonia. Blood cultures were collected and a trans-thoracic ecochacardiogram (TTE) was planned, in order to exclude endocarditis. The patient presented rapid defervescence, but remained asthenic; moreover CRP was $12 \mathrm{mg} / \mathrm{dL}$ after 6 days of antibiotic therapy. The patient underwent a TTE, which incidentally showed a 9-cm liver hypoechoic lesion. A thoracic/abdominal computed tomography (CT) scan revealed multiple bilateral nodular opacities in the lungs with peripheral cavitations that were consistent with septic emboli and a large abscess in the left lobe of the liver $(7 \times 9 \times 10 \mathrm{~cm})$ (Figures 1 and 2). No other radiological findings consistent with chronic lung disease or interstitial pneumonia were revealed. Blood cultures were positive for extended spectrum $\beta$-lactamase, negative for $K$. pneumoniae, therefore intravenous levofloxacin $750 \mathrm{mg} /$ die and imipenem/cilastatin 500/500 mg quater in die were started. The patient underwent the TEE, which showed a thickened ICD tip, strongly suggestive of electrocatheter endocarditis, and patent foramen ovalis (PFO) (Figure 3). Antibiotic therapy was maintained and the liver abscess was treated with percutaneous drainage. According to international guidelines, ${ }^{7}$ after 30 days of antimicrobial therapy, the ICD was re-

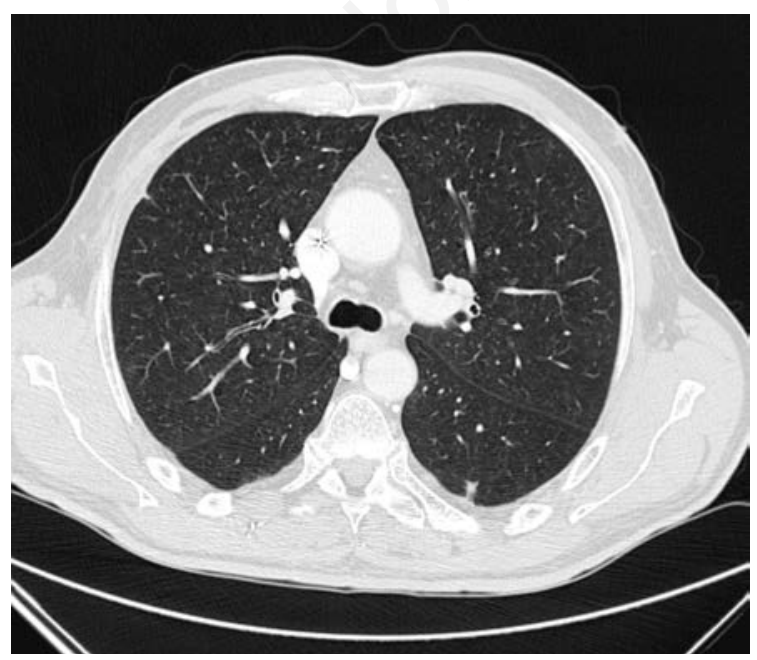

Figure 1. Multiple bilateral nodular opacities in the lungs with peripheral cavitations consistent with septic emboli (computed tomography scan). moved in a specialized center (Spedali Civili di Brescia) and re-implanted. At discharge, the blood test was within the normal range and the patient was asymptomatic. Radiological follow up showed improvement of lung and hepatic lesions.

\section{Discussion and Conclusions}

To our knowledge, this is the second reported case of $K$. pneumoniae liver abscess with multiple septic emboli associated with infective endocarditis.

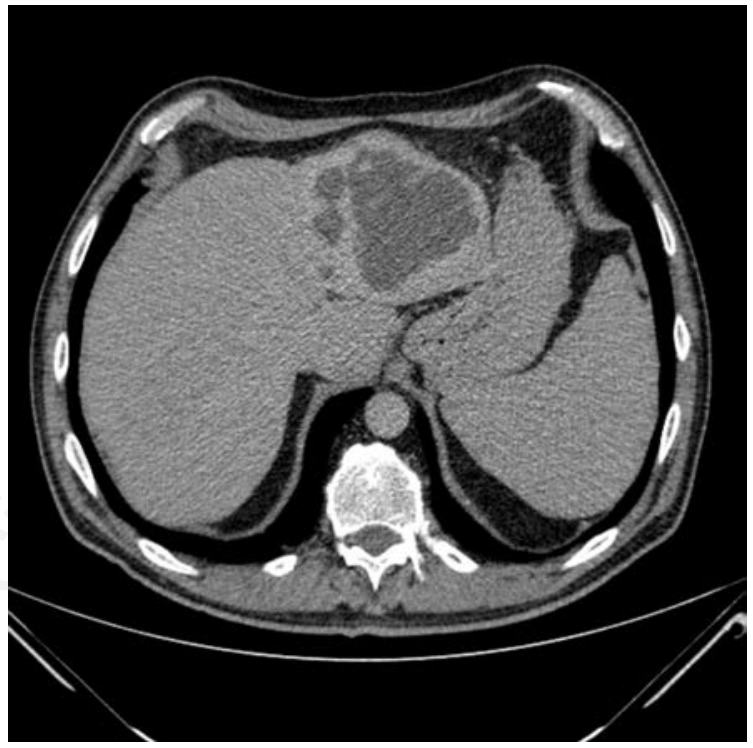

Figure 2. Abscess in the left lobe of the liver by computed tomography scan $(7 \times 9 \times 10 \mathrm{~cm})$.

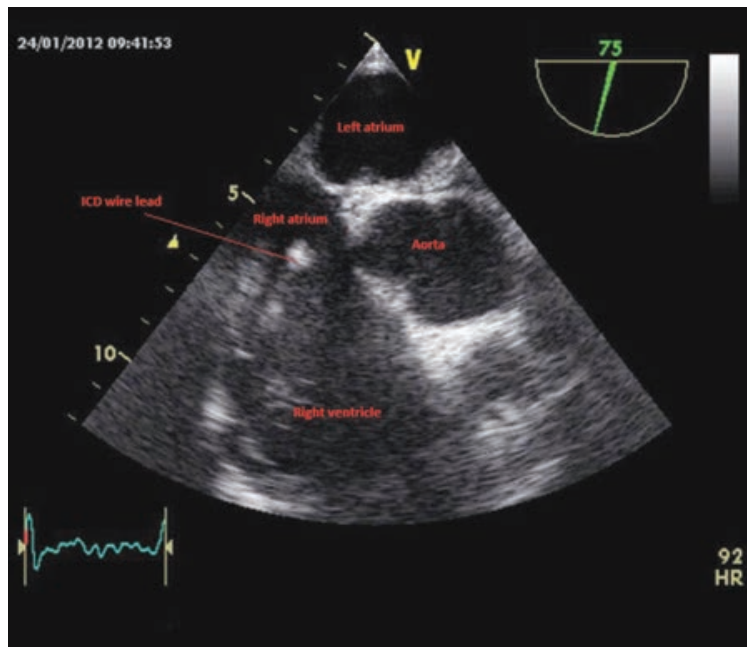

Figure 3. Trans-thoracic ecochacardiogram shows thickened implantable cardioverter defibrillator catheter strongly suggestive of electrocatheter endocarditis. 
Gram negative (including K. pneumoniae) infective endocarditis is rare (5\% of cases). The aortic valve is the most common involved site. The urinary tract infections and pacemaker infections are the most common source of bacteremia reported in the literature. ${ }^{5} \mathrm{In}$ the 1990s K. pneumoniae emerged as the leading etiological agent of community-acquired pyogenic liver abscess in East Asia. ${ }^{8}$ Several cases of K. pneumoniae liver abscesses have been recently reported outside Asia. ${ }^{9-11}$ In large case series of K. pneumoniae liver abscesses, between 40 and $78 \%$ of patients were affected by diabetes mellitus or impaired glucose tolerance. ${ }^{8}$ Retrospective studies on cardiac device-related endocarditis reported several risk factors for developing $\mathrm{CDE}$, which in all cases included diabetes mellitus. ${ }^{12}$ Besides fever, signs of CDE are signs of inflammation in the area surrounding the implanted cardiac device, leukocytosis, anemia and pulmonary emboli (asymptomatic in $40 \%$ of patients), if right heart endocarditis is present. Our patient was hospitalized for fever of unknown origin. The first findings were lung lobar infiltrates in the chest X-rays, which appeared as metastatic emboli only in the CT scan, performed when the liver abscess was diagnosed. No radiological findings consistent with chronic lung disease or interstitial pneumonia were found, leading us to assume that lungs were not the primary source of infection. The initial event was probably an ICD endocarditis with septic metastatic events in the liver and in the lungs through the PFO. The source of bacteremia could have been a urinary tract infection, as previously reported. ${ }^{13}$ Literature data show a significant delay between the onset and the diagnosis of device-related endocarditis (mean 5.5 months). ${ }^{12}$ Therefore, diagnosis of CDE should always be suspected in presence of fever after cardiac device insertion. Blood cultures and echocardiography should always be performed.

\section{References}

1. Osmonov D, Ozcan KS, Erdinler I, et al. Cardiac device-related endocarditis: 31-years' experience. J Cardiol 2013,61:175-80.
2. Catanchin A, Murdock CJ, Athan E. Pacemaker infections: a 10-year experience. Heart Lung Circ 2007;16: 434-9.

3. Del Rio A, Anguera I, Mirò JM, et al. Surgical treatment of pacemaker and defibrillator lead endocarditis: the impact of electrode lead extraction on outcome. Chest 2003;124:1451-9.

4. Viola G, Awan LL, Darouiche RO. Nonstaphylococcal infections of cardiac implantable electronic devices. Circulation 2010;121:2085-91.

5. Anderson MJ, Janoff EN. Klebsiella endocarditis: report of two cases and review. Clin Infect Dis 1998;26:468-74.

6. Rivero A, Gomez E, Alland D, et al. K2 serotype Klebsiella pneumoniae causing a liver abscess associated with infective endocarditis. J Clin Microbiol 2010;48: 639-41.

7. Baddour LM, Epstein AE, Erickson CC, et al. Update on cardiovascular implantable electronic device infections and their management: a scientific statement from the American Heart Association. Circulation 2010;121: 458-77.

8. Wang JH, Liu YC, Lee SS, et al. Primary liver abscess due to Klebsiella pneumoniae in Taiwan. Clin Infect Dis 1998;26:1434-8.

9. Garcia-Morillo JD, De la Cruz-Vincente F, Lopez-Ruiz T, Beranbeu-Wittel M. Hepatic abscess due to Klebsiella pneumoniae in an Asian immigrant. Enferm Infect Microbiol Clin 2006;24:585-6.

10. Sobirk SK, Struve C, Jacobson SG. Primary Klebsiella pneumoniae liver abscess with metastatic spread to lung and eye, a North European case report of an emerging syndrome. Open Microbiol J 2010;4:5-7.

11. Moore R, O'Shea D, Geoghegan T, et al. Communityacquired Klebsiella pneumoniae liver abscess: an emerging infection in Ireland and Europe. Infection 2013; 41:681-6.

12. Edelstein S, Yahalom M. Cardiac device-related endocarditis: epidemiology, pathogenesis, diagnosis and treatment - a review. Int J Angiol 2009;18:167-72.

13. Cheng DL, Liu YC, Yen MY, et al. Septic metastatic lesions of pyogenic liver abscess. Their association with Klebsiella pneumoniae bacteremia in diabetic patients. Arch Intern Med 1991;151:1557-9. 